\title{
Beam Damage During Energy-Dispersive X-ray Spectroscopy of FePt Nanoparticles
}

\author{
J. Bentley ${ }^{1}$, J. E. Wittig ${ }^{2}$ and J. R. McBride ${ }^{3}$ \\ ${ }^{1}$ Microscopy and Microanalytical Sciences, PO Box 7103, Oak Ridge, TN 37831-7103, USA \\ 2 Interdisciplinary Materials Science, Vanderbilt University, PMB 351683, Nashville, TN 37232, USA \\ ${ }^{3}$ Department of Chemistry, Vanderbilt University, PMB 351683, Nashville, TN 37232, USA
}

There remains much interest in the development of FePt for magnetic recording media. For almost a decade we have performed extensive studies of FePt multilayers and nanoparticles, especially epitactic growth on layers of $\mathrm{MgO}$ and the phase transformation from a disordered solid solution to the L10 ordered structure that exhibits attractive magnetic properties [1-7]. Many studies incorporated atomic-resolution high-angle annular dark-field (HAADF) scanning transmission electron microscope (STEM) imaging which readily discriminates the Pt and Fe because of the large difference in their atomic numbers. The studies at Oak Ridge National Laboratory also included in-situ annealing with a prototype Protochips Aduro heating holder in a probe-corrected JEOL 2200 operated at $200 \mathrm{kV}$. Complex annealing behavior was frequently observed including unexpected $\mathrm{L}_{2}$ ordering which implied a local composition nearer to Pt3Fe than FePt. It was unclear whether some of these observed effects were artifacts caused by the electron beam or were due to incorrect composition of as-synthesized nanoparticles. Some evidence of the latter was obtained but composition measurements by energy-dispersive X-ray spectrometry (EDS) in a Philips (FEI) CM200FEG with probes of $\sim 1 \mathrm{nA}$ were compromised by beam damage, frequently with a dramatic loss of Fe. This effect was somewhat unexpected because $200 \mathrm{kV}$ is well below the displacement threshold for Fe in FePt. At surfaces atomic bonding may be weaker but it is unknown if that is a major contribution to the responsible mechanism. Certainly, hole drilling at beam energies well below the (bulk) displacement threshold has been a commonly observed effect since soon after the introduction of FEG-STEMs and is frequently more pronounced in the presence of oxide on the specimen surface. Conventional wisdom is that surface carbon (contamination) is actually helpful in preventing hole drilling, perhaps as a sacrificial surface species that self-heals by surface diffusion as it is sputtered away. However, in the case of FePt nanoparticles deposited on a carbon support film it was postulated that, under the action of the electron beam, the carbon might react with the Fe exacerbating, if not directly causing, loss of Fe from FePt.

With the advent of more efficient EDS detectors or detector arrays that have almost an order-of-magnitude greater $\mathrm{SO}_{2}$ lid angle of collection than the EDS detectors that were typically found on TEMs and STEMs (30 $\mathrm{mm}^{2}$ and a collection angle of a little over $0.1 \mathrm{sr}$ - a specification that changed little for almost 4 decades!), an investigation has begun, not only to determine if the as-synthesized nanoparticle composition can be reliably measured, but also perhaps to reveal composition gradients within a nanoparticle, such as a coreshell structure. Certainly EDS spectrum images have successfully revealed such structures in other nanoparticles.

Backthinned plan-view specimens of complex, multilayer, developmental magnetic-recording media with $\sim 10 \mathrm{~nm}$ of FePt grown on a thin layer of $\mathrm{MgO}$ were characterized with an FEI ChemiSTEM ${ }^{\mathrm{TM}}$ Tecnai Osiris $^{\mathrm{T}}$ operated at $200 \mathrm{kV}$ at Vanderbilt University. This instrument is equipped with a Super-X ${ }^{\mathrm{TM}}$ array of 4 windowless silicon drift detectors (SDDs) with a specified collection angle of $0.9 \mathrm{sr}$, but has no EELS capability. Drift-corrected spectrum imaging was performed with both FEI (TIA) and Bruker Esprit (HyperMap) software. Spectrum imaging with TIA involves single EDS spectrum acquisition at each pixel, whereas Esprit employs repeated fast scans in a position-tagged spectrometry mode. Figure 1 shows Esprit 
HyperMap data for a $25 \times 25 \mathrm{~nm}$ area and acquisition time of $1250 \mathrm{~s}$ with a STEM probe of $<1 \mathrm{~nm}$ containing $2.5 \mathrm{nA}$. The surfaces of the nanoparticles are slightly deficient in Fe and the $\mathrm{MgO}$ appears mostly between the FePt particles. Figure 2 shows subsequently acquired data $(64 \mathrm{x} 64 \mathrm{~nm}, 300 \mathrm{~s}$ acquisition) approximately centered on the same region. The beam damage to the original $25 \mathrm{x} 25 \mathrm{~nm}$ area is evident in the ADF STEM image and the elemental maps. The loss of $\mathrm{MgO}$ and rearrangement of the FePt particles are particularly clear. Such specimen instability has to have contributed to degradation of the data of Figure 1 although it is masked in the integrated results. Similar behavior was found for spectrum imaging in TIA and Esprit with equivalent conditions (identically sized area, total acquisition time and probe current/size). By varying the probe current and dwell/acquisition times it appears that the beam damage is chiefly dependent on dose rather than dose rate. Further investigation of beam damage to FePt nanoparticles supported on thin carbon films is planned. The present preliminary data indicate that the more efficient EDS detector helps but care is still needed to avoid beam damage to the specimen during analysis [8].

\section{References}

1. J E Wittig, Y Fu, J Bentley, N D Evans \& B Lu, Microsc Microanal 11-Suppl 2 (2005) 1820

2. Y Fu, J E Wittig, J Bentley, N D Evans \& B Lu, Microsc Microanal 12-Suppl 2 (2006) 986

3. J E Wittig, J Bentley A Lupini \& C M Lukehart, Microsc Microanal 13-Suppl 2 (2007) 1196

4. J E Wittig, J Bentley L F Allard, M S Wellons and C M Lukehart, Microsc Microanal 14-Suppl 2 (2008) 216

5. J E Wittig, J Bentley L F Allard, M S Wellons and C M Lukehart, Microsc Microanal 14-Suppl 2 (2008) 348

6. J E Wittig, J Bentley L F Allard and C M Lukehart, Microsc Microanal 16-Suppl 2 (2010) 1754

7. J E Wittig \& L F Allard, Microsc Microanal 18-Suppl 2 (2012) 304

8. JB acknowledges his appointment as Adjoint Professor of Materials Science at Vanderbilt University in contributing to the performance of this work. JEW gratefully acknowledges support from Western Digital.
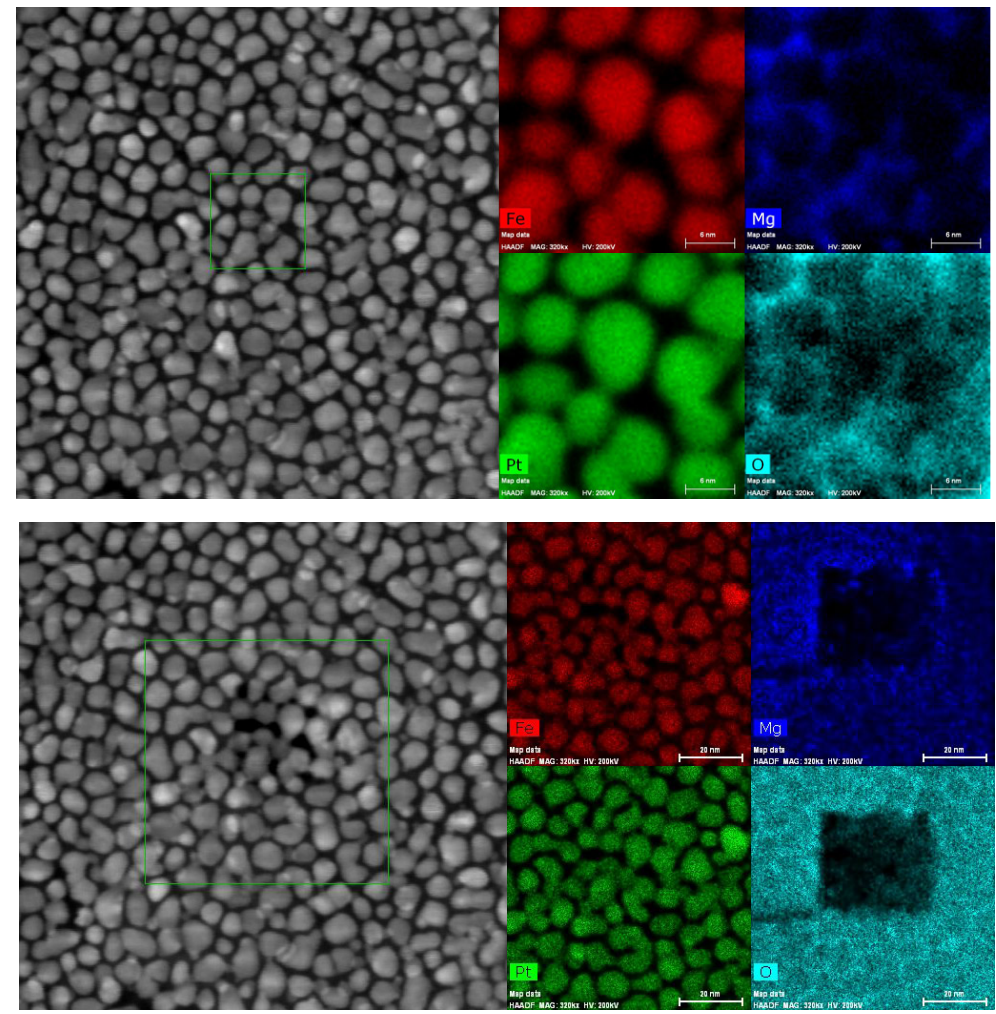

Figure 1. Green box on HAADF STEM image shows approximate position of $25 \times 25 \mathrm{~nm}$ area used for Esprit HyperMap spectrum image acquisition $(1250$ s) with $<1-n m$ probe of $2.5 \mathrm{nA}$. Elemental maps reveal nanoparticle surfaces are slightly deficient in $\mathrm{Fe}$ and $\mathrm{MgO}$ is mostly located between particles.

Figure 2. Green box on HAADF STEM image shows $64 \times 64 \mathrm{~nm}$ area used for Esprit HyperMap spectrum image acquisition (300 s) with $<1-\mathrm{nm}$ probe of $2.5 \mathrm{nA}$.. Elemental maps revea rearrangement of $\mathrm{FePt}$ nanoparticles and loss of $\mathrm{MgO}$ resulting from the acquisition of data for Figure 1. 